\title{
Quantifying impacts of the COVID-19 pandemic through life-expectancy losses: a population-level study of 29 countries
}

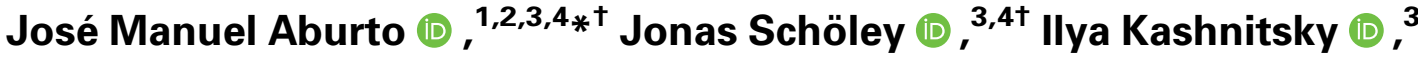

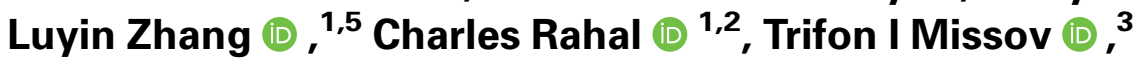 \\ Melinda C Mills $(\mathbb{D})$, ${ }^{1,2}$ Jennifer B Dowd (1) ${ }^{1,2}$ and Ridhi Kashyap (1) ${ }^{1,2+}$
}

${ }^{1}$ Leverhulme Centre for Demographic Science and Department of Sociology, University of Oxford, Oxford, UK, ${ }^{2}$ Nuffield College, Oxford, UK, ${ }^{3}$ Interdisciplinary Centre on Population Dynamics, University of Southern Denmark, Odense, Denmark, ${ }^{4}$ Laboratory of Population Health, Max Planck Institute for Demographic Research, Rostock, Germany and ${ }^{5}$ St Cross College, Oxford, UK

*Corresponding author. 42-43 Park End Street, Oxford, 0X1 1JD, UK.

E-mail: aburto@demogr.mpg.de; jose-manuel.aburto@sociology.ox.ac.uk

${ }^{\dagger}$ These authors contributed equally to this manuscript.

Editorial decision 6 September 2021; Accepted 8 September 2021

\begin{abstract}
Background: Variations in the age patterns and magnitudes of excess deaths, as well as differences in population sizes and age structures, make cross-national comparisons of the cumulative mortality impacts of the COVID-19 pandemic challenging. Life expectancy is a widely used indicator that provides a clear and cross-nationally comparable picture of the population-level impacts of the pandemic on mortality.

Methods: Life tables by sex were calculated for 29 countries, including most European countries, Chile and the USA, for 2015-2020. Life expectancy at birth and at age 60 years for 2020 were contextualized against recent trends between 2015 and 2019. Using decomposition techniques, we examined which specific age groups contributed to reductions in life expectancy in 2020 and to what extent reductions were attributable to official COVID-19 deaths.

Results: Life expectancy at birth declined from 2019 to 2020 in 27 out of 29 countries. Males in the USA and Lithuania experienced the largest losses in life expectancy at birth during 2020 ( 2.2 and 1.7 years, respectively), but reductions of more than an entire year were documented in 11 countries for males and 8 among females. Reductions were mostly attributable to increased mortality above age 60 years and to official COVID-19 deaths.

Conclusions: The COVID-19 pandemic triggered significant mortality increases in 2020 of a magnitude not witnessed since World War II in Western Europe or the breakup of the Soviet Union in Eastern Europe. Females from 15 countries and males from 10 ended up with lower life expectancy at birth in 2020 than in 2015.
\end{abstract}

Key words: COVID-19, demography, life expectancy, mortality 


\section{Key Messages}

- This is the first study to assemble a high-quality data set of harmonized mortality estimates, life tables and age by cause decomposition for 29 countries representing most of Europe, Chile and the USA to provide novel evidence of the cumulative, comparative impacts of the pandemic on population health.

- Out of 29 countries analysed, the COVID-19 pandemic led to losses in life expectancy in 27, with large losses of life expectancy of $>1$ year in 11 countries for males and 8 among females.

- Losses in life expectancy observed in Central and Eastern European countries in 2020 exceeded those observed around the dissolution of the Eastern Bloc (with the exception of Lithuania and Hungary), whereas similar magnitudes of losses in Western Europe were last seen around World War II.

- Compared against recent trends, females from 15 countries and males from 10 ended up with lower life expectancy at birth in 2020 than in 2015-a year when life expectancy was adversely impacted already due to an especially bad flu season.

- Losses in life expectancy were largely attributable to increased mortality above age 60 years and linked to official COVID-19 deaths.

\section{Introduction}

More than 1.8 million lives are estimated to have been lost due to COVID-19 around the world in 2020., ${ }^{1,2}$ This estimate-although staggering-masks the uneven impact of the pandemic across different countries and demographic characteristics like age and sex, ${ }^{3}$ as well as its impact on population health, years of life lost ${ }^{4}$ and longevity. ${ }^{5}$ Moreover, variations in testing capacity coupled with definitional inconsistencies in counting COVID-19 deaths make the true global toll of COVID-19 infections difficult to estimate with accuracy. ${ }^{6}$ To address these measurement challenges, significant efforts have been directed at the harmonization and analysis of all-cause mortality data. A widely used approach to quantify the burden of the pandemic using allcause mortality is through the analysis of excess mortality, defined as the number of deaths observed during the pandemic above a baseline of recent trends. ${ }^{5,6}$ Here we go beyond excess deaths and country-specific analyses and focus on the pressing issue of revealing the impacts of the pandemic on life expectancy from a cross-national perspective.

Life expectancy at birth is the most widely used metric of population health and longevity. It refers to the average number of years a synthetic cohort of newborns would live if they were to experience the death rates observed in a given period throughout their lifespan. This indicator is thus often referred to as 'period life expectancy', as it simulates and summarizes the implications of a mortality profile from a calendar year. Although the indicator does not describe the actual life course of a cohort ${ }^{7}$ and should not be interpreted as a projection or forecast of any individual's lifespan, ${ }^{8,9}$ it provides a timely description of current mortality patterns. The key advantage of period life expectancy arises from the fact that it is age-standardized, making it the preferred indicator for comparisons across countries with populations of different sizes and age structures, and over time. ${ }^{9}$ Life expectancy can also be calculated as conditional on surviving to a given age, e.g. 60 years, where it refers to remaining life expectancy from age 60 years. The study of life expectancy in the context of the COVID-19 pandemic matters because it enables us to compare the cumulative impacts of the pandemic against past mortality shocks and recent trends across different countries using a standardized indicator that is routinely monitored to capture differences in mortality.

Prior to the pandemic, life expectancy at birth typically increased almost monotonically in most countries over the twentieth and into the twenty-first centuries. ${ }^{10}$ In recent decades, improvements in life expectancy among high-income countries were predominantly driven by gains made at older ages ( $\geq 65$ years), ${ }^{11}$ although significant crosscountry heterogeneity persists. This heterogeneity has become more prominent since 2010. Whereas some countries in Eastern Europe and the Baltics experienced significant gains in life expectancy in the past decade, ${ }^{12}$ others witnessed noticeable slowdowns in the pace of improvements and, in some cases, stalls or even temporary reversal. ${ }^{13}$ For example, life expectancy in the USA, ${ }^{14}$ England \& Wales and Scotland saw only limited gains in the last decade. ${ }^{15,16}$ These atypical trends have been linked to slower improvements in old-age mortality and increases in working-age death rates. ${ }^{15}$ 
In a context in which trajectories of life-expectancy progress became more varied, the COVID-19 pandemic triggered a global mortality crisis posing additional challenges on population health. Death rates from COVID-19 tend to be higher among males than females, with higher case-fatality rates among older age groups ${ }^{17,18}$ - precisely those that have accounted for mortality improvements in recent years. The pandemic also indirectly affected mortality from other causes of death. Emerging evidence has highlighted the impacts of delayed treatments or avoidance of care-seeking for cancers or cardiovascular diseases ${ }^{19,20}$ resulting in increased mortality from these conditions, whereas lockdowns may have reduced the number of deaths due to accidents. ${ }^{21}$

This study is the first to use an unprecedented collection of demographic data from 29 countries, representing most of Europe, Chile and the USA, to examine the impacts of the pandemic on life expectancy in 2020, contextualized against trends in 2015-2019. To enable reliable crossnational comparisons of life expectancy over the period 2015-2020, we harmonized death counts and population estimates from multiple sources, leveraging major ongoing efforts collecting data on all-cause mortality ${ }^{22}$ and official COVID-19 deaths..$^{23}$ An interactive dashboard that accompanies our manuscript is available at https://covid19.demo graphicscience.ox.ac.uk/lifeexpectancy. Only countries with high-quality age-disaggregated all-cause mortality data were included to estimate life tables (see the 'Methods' section). We focus on the pressing questions of how much life expectancy changed in 2020 relative to the period 2015-2019 and whether the impact was different for males and females. Leveraging demographic decomposition methods, we examine which age groups contributed to changes in life expectancy in 2020 and to what extent the observed reductions in life expectancy were attributable to officially reported COVID-19 deaths.

\section{Methods}

\section{Data}

All-cause death counts were retrieved from the Short Term Mortality Fluctuations (STMF) data series within the Human Mortality Database (HMD). ${ }^{22}$ Additional allcause mortality data were retrieved for the USA from the Centers for Disease Control and Prevention $(\mathrm{CDC})^{24}$ and England \& Wales from the Office for National Statistics $(\mathrm{ONS})^{25}$ to supplement the STMF data. The STMF provides high-quality weekly death counts for 38 countries (at the time of writing) in both a harmonized and original format, but the completeness of these data varies by country. We processed the original input files and selected
29 countries for further analysis. We selected only those countries for which sex-specific death counts across at least 10 age groups were available for every week in 2020 (see Supplementary Table S1, available as Supplementary data at IJE online). Death counts for the USA and England \& Wales across the years 2015-2019 were only reported in extremely coarse age groups by the STMF, but were available in fine grouping for 2020. For both countries, we therefore utilized age-specific annual death counts as reported by the $\mathrm{CDC}^{24}$ and the $\mathrm{ONS}^{25}$ for the years prior to 2020. All-cause mortality data were supplemented with COVID-19 deaths from the COVerAGE-DB database for a subset of 18 countries (by sex) registered continuously since March 2020 until the end of the year. ${ }^{23}$ COVerAGEDB provides information about COVID-19 confirmed cases and deaths as reported by statistical agencies for $>100$ countries, in a standardized format with harmonized age groups. Population estimates were retrieved from the UN for the years 2015-2020; and for the year 2020, we used age-specific mid-year population projections from World Population Prospects 2019 (WPP). ${ }^{10}$ For England $\&$ Wales, Northern Ireland and Scotland, mid-year population estimates from HMD for the year 2018 were projected for 2019 and 2020 using stable population assumptions (see Supplementary Figure S1, available as Supplementary data at IJE online). We opted against official projections because the WPP does not disaggregate the UK into its constituent countries and the oldest age group in ONS projections is $\geq 90$ years.

\section{Mortality estimation}

All-cause mortality data are available in irregular age groups for different countries. We harmonized data from the STMF, CDC, ONS and COVerAGE-DB using a penalized composite link model (PCLM) that estimated death counts in single years of age from 0 to 110 years from the grouped data. ${ }^{26,27}$ The PCLM model was fitted independently to each country, sex and year combination, and the smoothing parameters were estimated via a Bayesian Information Criteria-based grid search. The data points are death counts by age group within each year, country and sex (at least 10 data points for each population). Prior to ungrouping, we summed the weekly death counts from the STMF data into annual death counts, also taking into account deaths reported in unknown weeks. In cases in which the age grouping changed from one week to the next, we first summed all the deaths within a stratum and year belonging to the same age-grouping scheme, applied PCLM ungrouping separately for each scheme and then summed the ungrouped deaths into annual counts by single years of age. 
Person-years of exposure were approximated by estimated or projected mid-year population counts and used as the denominator of the age-specific death rates. Exposure estimates were adjusted for leap-weeks, as most countries in the STMF data report deaths using the ISO week date calendar. ${ }^{28}$ The length of a year in the ISO week calendar is either 371 days in a leap-week year such as 2020 or 364 days in a regular year such as 2019 . Thus, the longer reporting interval for leap-week years would, all things being equal, increase observed death counts by a factor of $371 / 364=1.9 \%$ over regular years. To counter this spurious effect, we adjusted the exposures by the factor 371/364 in ISO week date leap years. This adjustment has been made for all countries except for annual deaths prior to 2020 for the USA and England \& Wales, which are reported over a Gregorian year by the CDC and ONS sources.

\section{Life-table construction}

Life tables for all 29 countries by sex for the period 20152020 were constructed following a piece-wise constant hazard model using all-cause mortality by single age with the last age interval grouping deaths at ages $\geq 85$ years, consistently with standard demographic techniques. ${ }^{29}$ Our choice of grouping deaths of age $\geq 85$ years is common practice in demographic research and it ensures comparability across countries, especially because some countries used this last age group to report deaths for 2020. The choice of the last open-ended interval may impact estimates of life expectancy depending on the proportion of the population surviving to this last interval. ${ }^{30}$ To evaluate its impact, we further calculated life tables by grouping death counts estimated from the PCLM model age $\geq 100$ years for countries where more granular age categorization was available (see Supplementary Figure S2, available as Supplementary data at IJE online). Smaller populations were more sensitive to the choice of the last open-ended group. However, trends in life expectancy remained similar. From these life tables, life expectancy at birth and life expectancy conditional on surviving to age 60 years for males and females were extracted for each country by sex. We performed several sensitivity checks to ensure the robustness of our estimates including comparisons with alternative sources such as the UN and from the HMD for the period from 2015 to 2019, or their most recent year available (see Supplementary Figure S2, available as Supplementary data at IJE online). Additionally, we compared full-year death counts in our estimates with those reported in the HMD for previous years (Supplementary Figure S3, available as Supplementary data at IJE online). Most countries did not show deviations across sources, except for Austria, where the STMF underestimates death counts varying from $2 \%$ and $4 \%$ for females and males, respectively. We further calculated life expectancy using the death counts from both sources and WPP population estimates as exposures and found that the effect on life expectancy is negligible $(<1 \%)$. For the USA, we also compared our life-expectancy estimates with those based on provisional data up to June $2020 .^{31}$ Our results show a sharper decrease in life expectancy than those based on mid-year estimates, in line with including the remaining months of 2020 that also saw persistent levels of excess mortality.

\section{Confidence intervals}

We performed Poisson sampling of age-specific death counts to generate confidence intervals around the change in life expectancy from 2019 to $2020 .^{32}$ For each country, sex, year and age, we sampled 500 death counts from a Poisson distribution with a rate parameter equal to the expected death count predicted by the PCLM method. We calculated a complete life table for each of these 500 replications and derived the annual changes in life expectancy. The sampling distribution of these life-expectancy changes was summarized by the $2.5 \%$ and $97.5 \%$ quantiles, forming a $95 \%$ confidence interval.

\section{Decomposition of life expectancy by age and cause of death}

To disentangle age-specific effects and to quantify the impact of COVID-19 deaths on changes in life expectancy, we used the linear integral decomposition $\operatorname{method}^{33}$-a state-of-the-art method that allows us to decompose the difference of two values of life expectancy by age and cause of death, which has been implemented previously for this type of analysis. ${ }^{12,34}$ This methodology assumes that causes of death are exhaustive and independent, not allowing mortality to be duplicated. This assumption may not be realistic in the context of COVID-19, as the pandemic may have indirectly affected other causes of death. However, previous evidence suggests that the net effect of interactions between causes of death are negligible in decomposition analysis. ${ }^{35}$ Moreover, our results are likely to be unaffected by this assumption if COVID-19 deaths are considered 'excess'. In addition, in most countries, the contribution of official COVID-19 deaths to changes in life expectancy can be interpreted as a lower bound due to limited testing and the potential misclassification of COVID-19 deaths. An alternative to decomposition methods is to quantify 'Years of Life Lost' defined as the difference between an individual's age at death from COVID-19 
and their remaining life expectancy at that age had the pandemic not occured. ${ }^{4}$ However, this measure of life lost is affected by population age structure and therefore comparisons between countries and over time are not precise. We decompose yearly changes in life expectancy by age and official COVID-19 deaths vs other remaining causes of death for each country by sex. This methodology has the advantage that the resulting estimates are additive and the total change in a given period-such as 2015-2019-is the sum of yearly estimates from 2015 to 2019. As part of our sensitivity checks, we replicated our results with the stepwise decomposition method ${ }^{36}$ and quantified the impact of COVID-19 deaths on life expectancy by calculating causedeleted life tables. ${ }^{29}$ Results from these analyses with respect to those based on the linear integral model were consistent.

\section{Results}

In our data set, life expectancy at birth among females in 2019 ranged from 78.6 years in Bulgaria to 86.5 years in Spain (Figure 1). Among males, it ranged from 71.4 years in Lithuania to 82.2 years in Switzerland. At age 60 years, countries in Eastern Europe and Scotland exhibited the lowest remaining life expectancy, whereas older females in France and Spain enjoyed the highest. Female life expectancy was larger than that of males in every country.

From 2015 to 2019, all countries experienced increases in life expectancy at birth, albeit with varying magnitudes (Figure 2). Among females, gains ranged from $\sim 1$ month per year in Greece, France and Scotland, to $>3$ months in Spain, Hungary and Lithuania. Among males, the lowest gains in life expectancy at birth were seen in the USA, Scotland, and Iceland (up to $\sim 1$ month per year), whereas Lithuanian males benefited from $>5$ months per year of additional life expectancy. Similar trends across countries were observed for life expectancy at age 60 years, emphasizing the importance of improvements in older-age survival.

In contrast, life expectancy declined in all countries for both sexes from 2019 to 2020, except for females in Finland and both sexes in Denmark and Norway (Figure 2). With the exception of Spain, Slovenia, Estonia and Northern Ireland, life-expectancy losses were larger

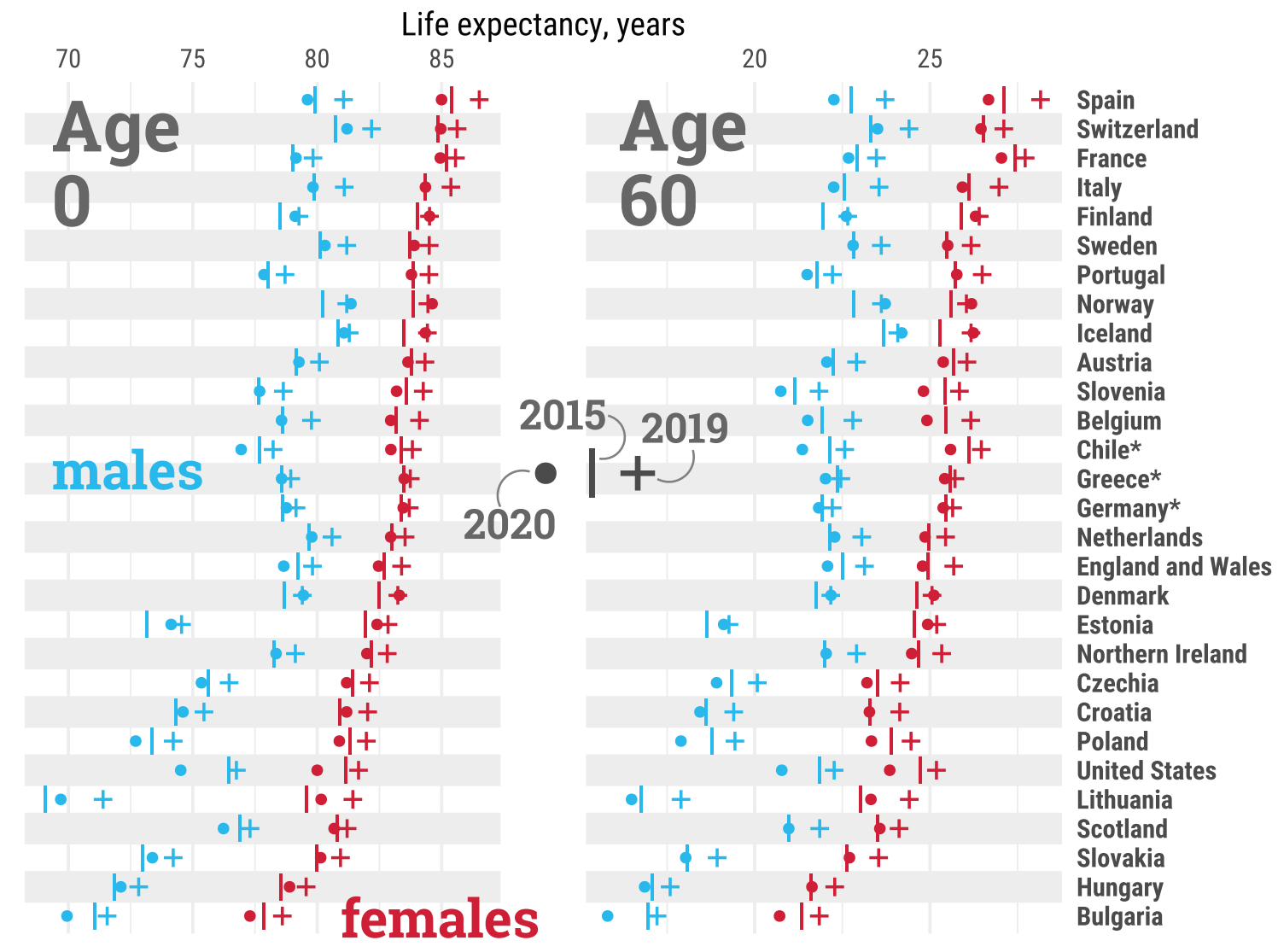

Figure 1 Life expectancy at birth (age 0, left panel) and at age 60 years (right panel) by country and sex, in 2015, 2019 and 2020 . Estimates for females (red), males (blue), $2015(\mid), 2019(+), 2020$ (O). Countries are sorted from highest to lowest levels of female life expectancy at birth in 2019. *Estimates for Chile, Greece and Germany were available from 2016. All data points are provided in a table in Supplementary File 2, available as Supplementary data at IJE online. An interactive version of this visualization is available at https://covid19.demographicscience.ox.ac.uk/ lifeexpectancy. 


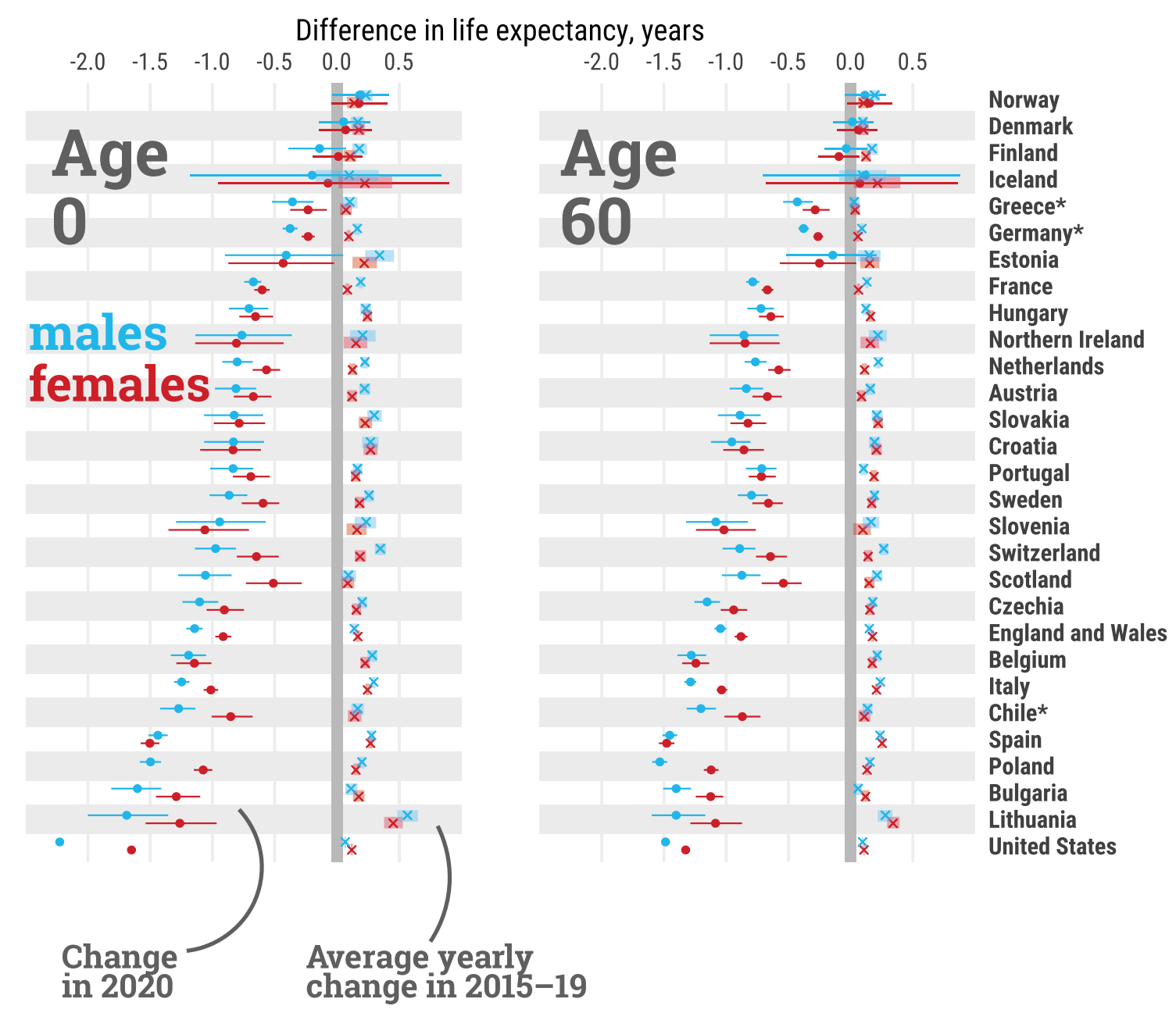

Figure 2 Average per-year change in life expectancy at birth (age 0) and age 60 years, by country and sex, from 2015 to 2019 , and total change from 2019 to 2020. Estimates for females (red), males (blue), average per-year changes from 2015 to 2019 are depicted by the symbol (x), dots depict the total change from 2019 to 2020. Lines represent $95 \%$ confidence intervals. Countries are sorted from smallest to largest losses between 2019 and 2020 in male life expectancy at birth. *Estimates for Chile, Greece and Germany were available from 2016. All data points are provided in a table in Supplementary File 2, available as Supplementary data at IJE online. An interactive version of this visualization is available at https://covid19.demo graphicscience.ox.ac.uk/lifeexpectancy.

for males in 2020. The magnitude of these declines offset most gains in life expectancy in the 5 years prior to the pandemic. Out of 29 countries, females from 15 and males from 10 countries ended up with lower life expectancy at birth in 2020 than in 2015, which was already an exceptionally bad year for life expectancy due to an irregularly strong flu season. ${ }^{13}$ Our results show that from 2019 to 2020 , females in 8 countries and males in 11 lost $>1$ year of life expectancy at birth. As Figure 3 shows, the magnitudes of losses observed in 2020 have not been witnessed since World War II in many countries of Western Europe such as Spain, England \& Wales, Italy, Belgium, France, the Netherlands, Sweden, Switzerland and Portugal, with data available for the full twentieth century (see also Supplementary Figure S4, available as Supplementary data at $I J E$ online for females). With the exception of Lithuania and Hungary, the losses observed in 2020 in Eastern
European countries exceeded those observed after the dissolution of the Eastern Bloc. The biggest losses of 1.5 years or more of life expectancy at birth were documented among males in the USA, Lithuania, Bulgaria and Poland, and females in the USA and Spain. At age 60 years, the three countries with the largest losses in male remaining life expectancy were Poland, the USA and Spain, each losing $>1.4$ years. Remaining life expectancy for females in Spain, USA and Belgium declined by $>1.2$ years. Note that annual changes in life expectancy are subject to random fluctuations that are larger for smaller populations. Thus, the ranking of the countries is not only affected by the pandemic-induced mortality shock, but also by the inherent randomness of death counts, which are more sensitive for smaller populations. This is indicated in Figure 2 via $95 \%$ confidence intervals around the estimated drop in life expectancy. 

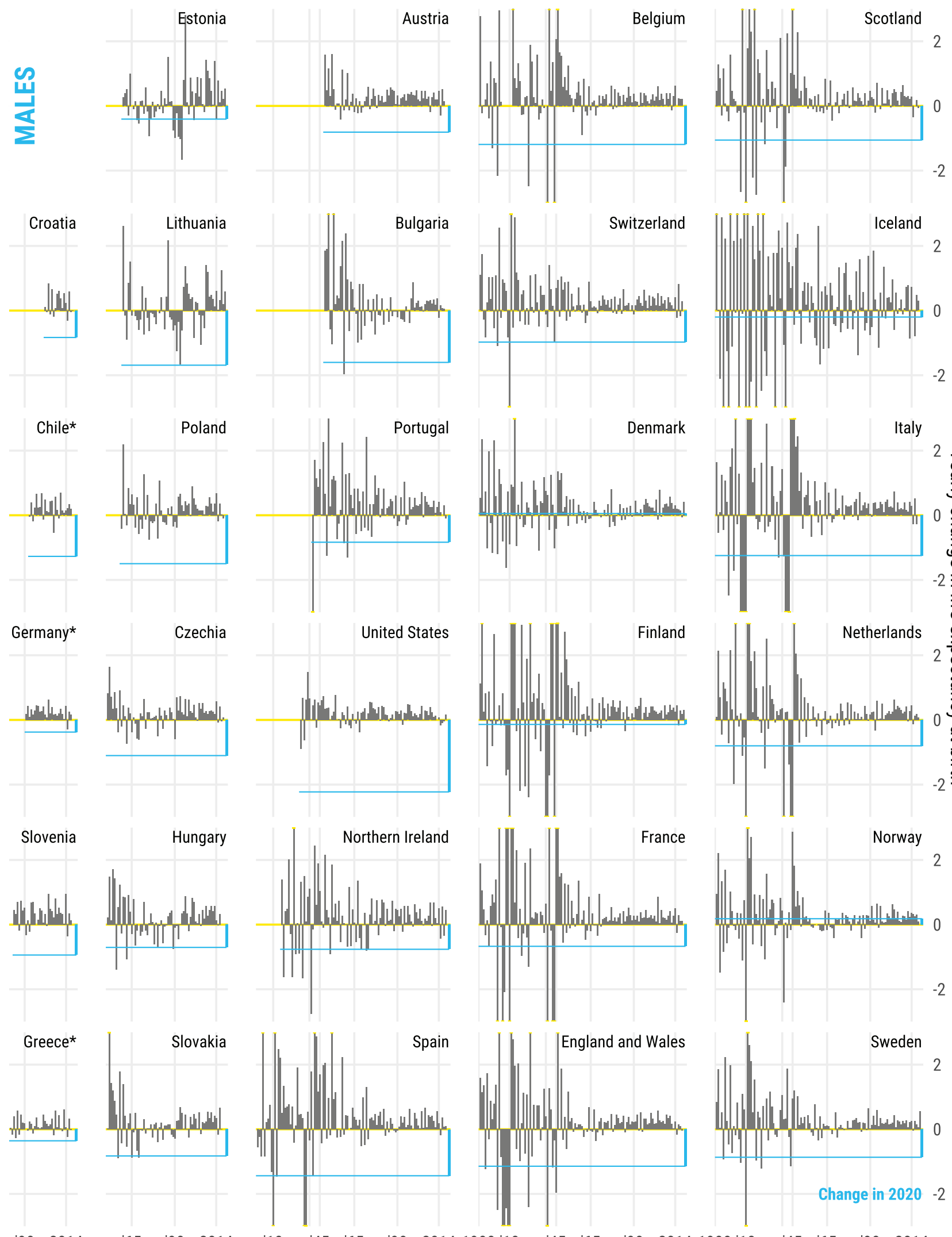

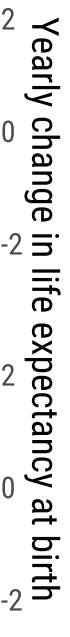

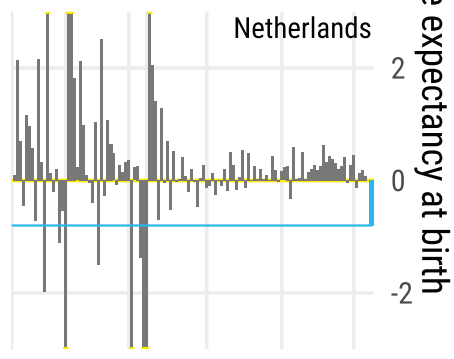

'90 $2014 \quad 65 \quad 90 \quad 2014$

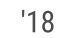

5 '65 '90 $20141900 ' 18$
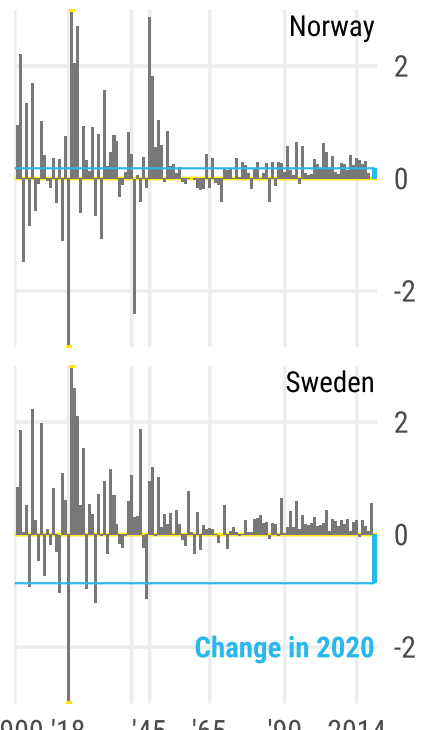

Figure 3 Annual change in male life expectancy at birth since 1900 or earliest year available in the Human Mortality Database for males. The straight blue line indicates the change from 2019 to 2020 , with the bars representing year-on-year change. 
Mortality reductions are translated into gains in life expectancy at birth and can be attributed to specific age groups. Bars shown in Figure 4 indicate the contributions, in years, to changes in life expectancy in 2015-2019 and 2019-2020 by broad age groups and sex (finer age grouping in Supplementary Figure S5, available as Supplementary data at IJE online). Between 2015 and 2019 , mortality improvements in those aged $\geq 60$ years consistently contributed to increasing life expectancy across countries. In several countries, life-expectancy gains in those aged $>80$ years surpassed those in the age group 60-79 years. For females in particular, mortality improvements in the $\geq 80$-years age group contributed most to improvements in life expectancy between 2015 and 2019, whereas for men broadly mortality improvements in the 60- to 79-years age group were greater. Improvements below age 60 years contributed less, but progress was still observed in many countries, especially in Eastern Europe, except for the USA and Scotland, where life expectancy decreased due to worsening mortality at these ages.

The COVID-19 pandemic led to sharp declines in life expectancy (see Figures 2 and 3), predominantly due to elevated mortality in the older age groups (see Figure 4). Increased death rates above age 60 years contributed the most to life-expectancy declines across all countries between 2019 and 2020. Increased death rates at ages $\geq 80$ years contributed most to life-expectancy losses among females. Among males, elevated death rates at ages 60-79 years contributed most to life-expectancy losses across many countries, but the impact of midlife mortality below age 60 years was noticeable too, especially those suffering the biggest losses in life expectancy such as the USA, Lithuania and Bulgaria. Notably increased midlife mortality $(0-59$ years) was the largest contributor

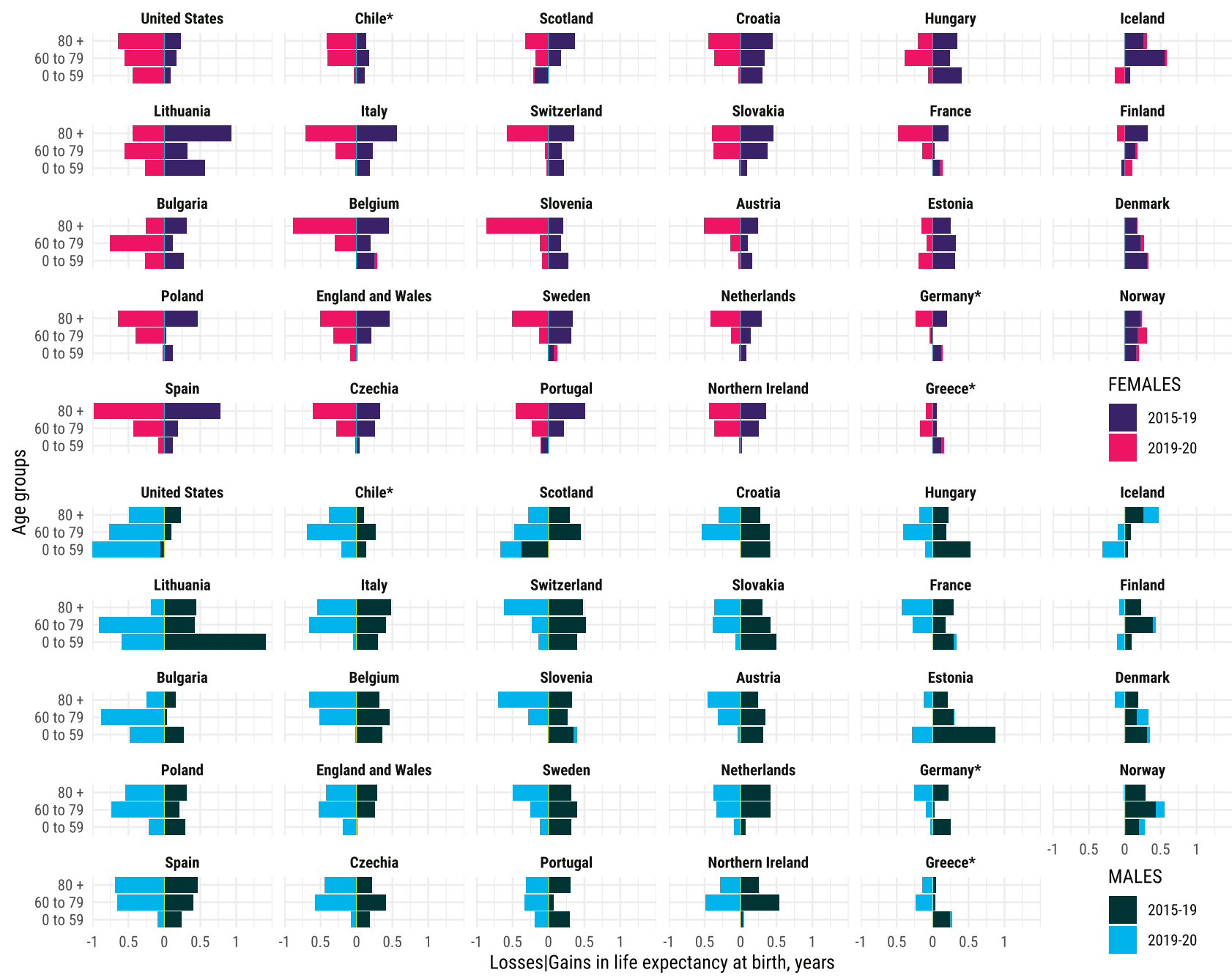

Figure 4 Contributions (in years) to changes in life expectancy at birth from 2015 to 2019, and from 2019 to 2020, attributable to mortality at age $<60$, $60-80$ and $>80$ years by country and sex. Positive values indicate gains in life expectancy; negative values indicate reductions in life expectancy. The sum of contributions over age results in the total change in life expectancy at birth during the specific period. Countries are sorted (column-wise) from largest to smallest losses between 2019 and 2020 in male life expectancy at birth. *Estimates for Chile, Greece and Germany were available from 2016. All data points are provided in a table in Supplementary File 3, available as Supplementary data at IJE online. 
to life-expectancy losses between 2019 and 2020 in the USA among males.

We were able to quantify the contribution of official COVID-19 deaths to life-expectancy reductions for 18 countries (Figure 5) for which more reliable age and sex-disaggregated data on COVID-19 deaths were available (see the 'Methods' section). We estimated that deaths reported as COVID-19 in the COVerAGE database ${ }^{23}$ explain most life-expectancy losses observed. Both females and males in Chile, England \& Wales, Belgium, Spain and Slovenia, and males in Italy experienced losses of more than a whole year of life expectancy at birth attributable to official COVID-19 deaths, explaining most of the overall loss between 2019 and 2020. In some contexts, such as Denmark and Chile, life-expectancy losses due to COVID-19 deaths were larger than total life-expectancy losses, as increased mortality due to COVID-19 was offset by mortality reductions among other causes.

\section{Limitations}

Although most of the countries included in our analysis have high-quality systems of death registration and usually report timely and complete death counts, ${ }^{37}$ there are likely issues related to delays in death registration in 2020. We deal with these potential issues by harmonizing and smoothing death counts with a PCLM that has been shown to provide reliable estimates of mortality for all countries in our study ${ }^{26}$ and by adjusting the person-years exposed. In most STMF countries, deaths that take place in a given week are covered by $>90 \%$ by the following two weeks (STMF metadata). Therefore, we expect the percentage of missing deaths in the last weeks of 2020 to be low. Moreover, according to the STMF metadata, some countries (such as Austria, England \& Wales, Germany and the Netherlands) employ extrapolation procedures to correct for incompleteness to adjust for missing death counts in the preceding 1-3 weeks. We recognize nonetheless that late and/or under-registration may affect our estimates by underestimating losses in 2020. Therefore, our results should be interpreted as estimates of the lower bound of life-expectancy reductions in 2020. For official COVID-19 mortality figures, there are likely to be inaccuracies due to differences in classification and testing practices across countries. Although we are unable to precisely measure the extent to which these inaccuracies may affect our estimates to make adjustments (i.e. since misclassification can occur for different reasons), we err on the side of caution and interpret our results as a lower bound of the impact of COVID-19 deaths on changes in life expectancy.

The magnitude of life-expectancy losses can also be influenced by migration due to COVID-19, especially in countries where persistent outmigration flows are not tracked accurately (such as via population registers) between censuses. For example, in Eastern European countries with high levels of outmigration in their populations, increased mortality could have occurred because of elevated return migration due to the COVID-19 pandemic. Nevertheless, we anticipate that the magnitude of these effects is likely to be small due to the broadly younger age

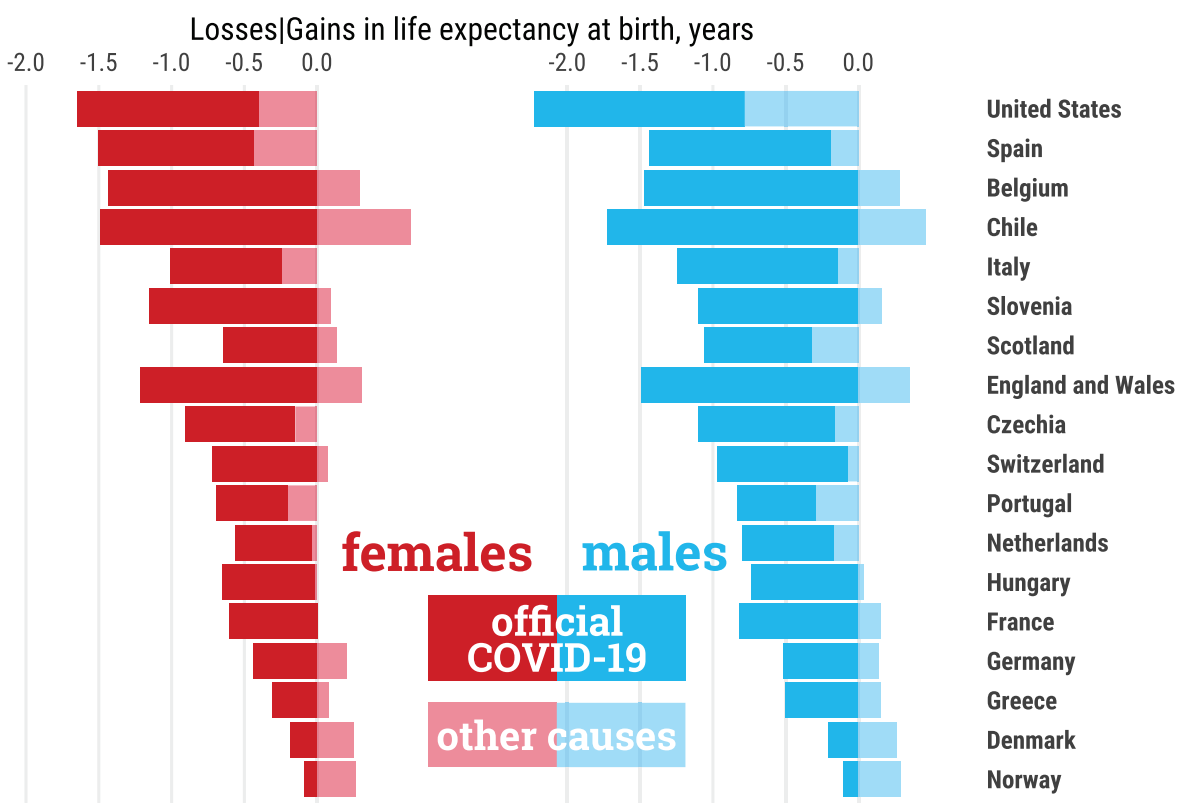

Figure 5 Contributions (in years) to changes in life expectancy at birth from 2019 to 2020 attributable to official COVID-19 deaths and remaining causes of death. Countries are sorted from largest to smallest losses. The sum of both components adds to the total change from 2019 to 2020 in a given country. All data points are provided in a table in Supplementary File 3, available as Supplementary data at IJE online . 
profiles of migrants and previous evidence that suggests that mortality at younger ages is more likely to be affected by international migration. ${ }^{38,39}$ In 2020 , as increased mortality occurred mostly at older ages, the effect of migration on our estimates is likely to be low.

\section{Discussion and conclusion}

Our analyses of life expectancy show that the pandemic exacted a striking toll on population health in 2020 across most of Europe, the USA and Chile. Only males and females in Denmark and Norway, and females in Finland were successful in avoiding drops in life expectancy in our cross-national comparison of 29 countries. Early nonpharmaceutical interventions coupled with a strong healthcare system may help to explain some of this success. ${ }^{40,41}$ In contrast, the USA, followed by Eastern European countries such as Lithuania, Bulgaria and Poland, experienced the largest losses in life expectancy in 2020, with larger losses in most countries for males than females. Among Western European countries, life-expectancy losses were also documented. To put this into perspective, the 20142015 strong flu season was regarded as an exceptionally bad year among high-income countries, causing drops in life expectancy. ${ }^{13}$ The largest drop was observed in Italy with a loss of around half a year among males and females. Our study reveals that 22 countries included in our study experienced larger losses than half a year in 2020. Females in 8 countries and males in 11 countries experienced losses of $>1$ year. To contextualize, it took on average 5.6 years for these countries to achieve a 1-year increase in life expectancy recently: progress wiped out over the course of 2020 by COVID-19. For Western European countries such as Spain, England \& Wales, Italy and Belgium, among others, the last time such large magnitudes of losses were observed in a single year was during World War II.

Whereas in Europe, increased mortality in the $\geq 60$ years age groups contributed most to life-expectancy losses, the pandemic saw a worsening of working-age mortality ( $<60$ years) in the USA, and this age group contributed most to life-expectancy losses for males. Despite having a younger population, the USA also has higher comorbidities in these age groups compared with European populations with greater vulnerability to COVID-19. ${ }^{42,43}$ Other factors, such as those linked to unevenness in healthcare access in the working-age population and structural racism, may also help to explain the increased mortality. Recent research from the USA, for example, shows that socially disadvantaged populations such as Blacks and Latinos experienced losses three times higher than those reported here at the national level. ${ }^{9}$ Emerging evidence further indicates that non-COVID-19 excess mortality was concentrated in working ages. ${ }^{44,45}$ Although some of this likely reflects misclassification of COVID-19 deaths due to factors linked to poorer testing and healthcare access, our findings reflect a deepening during the pandemic of the US mid-life mortality crisis that has led to the stalling of life expectancy in recent years. ${ }^{13,14,46}$

Countries in Eastern Europe (Lithuania, Bulgaria and Poland), as well as England \& Wales and Scotland, where recent life-expectancy stalls due to deteriorating midlife mortality too have been noted, also saw increased mortality in the $<60$-years age group. In the Eastern European countries, increased mortality in the $60-$ to 79 -years age group contributed most to life-expectancy losses in comparison with Western European countries such as Spain, Italy and Belgium, where increased mortality in the $\geq 80$-years age groups had sizable impacts. These patterns may reflect the continued impacts of the atypical mortality transitions and a delayed cardiovascular revolution in Eastern countries compared with Western Europe, ${ }^{12}$ whereby greater co-morbidities and vulnerabilities in population health are present at these ages.

Emerging evidence from low- and middle-income countries (such as Brazil and Mexico) that have been devastated by the pandemic ${ }^{6}$ suggests that life-expectancy losses may be even larger in these populations. Losses in life expectancy are also likely to vary substantially between subgroups within countries. However, a lack of data currently limits direct and more disaggregated comparisons across a wider range of countries, but these are urgently needed to understand the full mortality impacts of the pandemic. This limitation is manifested in our study as we were only able to include two countries outside of Europe with reliable and complete information for 2020, and our analysis focused on national populations. To expand our analyses to a broader set of countries, data on all-cause mortality with detailed disaggregation by age and sex, and for deeper analysis other characteristics such as socio-economic status and ethnicity, are needed to assess the uneven mortality impacts of the pandemic. Nevertheless, we note that in settings with deficient or limited vital registration, the true mortality impacts of the pandemic may never fully be known and, at minimum, the use of data from such contexts would require further adjustments (e.g. for undercounting, age heaping) before use. Although COVID-19 might be seen as a transient shock to life expectancy, the evidence of potential long-term morbidity due to long COVID and impacts of delayed care for other illnesses ${ }^{19,20}$ as well as health effects and widening inequalities stemming from the social and economic disruption of the pandemic $^{47}$ suggest that the scars of the COVID-19 pandemic on population health may be longer-lasting. 


\section{Supplementary data}

Supplementary data are available at IJE online.

\section{Ethics approval}

This research project does not require ethics approval as it uses only macro data that are freely available online.

\section{Funding}

British Academy's Newton International Fellowship grant NIFBA19/190679 (J.M.A., R.K.); ROCKWOOL Foundation's Excess Deaths grant (J.M.A., J.S., T.I.M., I.K.); Leverhulme Trust Large Centre Grant (J.M.A., L.Z., R.K., C.R., J.B.D., M.C.M.); John Fell Fund grant 0009182 (J.M.A., R.K., C.R., J.B.D., M.C.M.); European Research Council grant 835079 (M.C.M .).

\section{Data Availability}

The replication files for this paper include customized functionality written in the $\mathrm{R}$ statistical programming language. ${ }^{48}$ The code, and all harmonized input and output data pertaining to our analysis, is hosted both on Zenodo (a general-purpose open-access repository developed under the European OpenAIRE programme and operated by CERN) at https://zenodo.org/record/4556982 and on GitHub (https://github.com/oxforddemsci/ex2020).

\section{Acknowledgements}

We thank Alyson van Raalte, Jim Oeppen, Christiaan Monden and John Ermisch for their insightful comments on the manuscript. We also thank Ainhoa Alustiza for her timely response on issues related to the STMF data set.

\section{Author Contributions}

Conceptualization: J.M.A., J.S., R.K. Data curation: J.S., L.Z., J.M.A., R.K., T.I.M., I.K. Formal analysis: J.M.A., J.S., L.Z., I.K. Methodology: J.M.A., R.K., J.S., I.K. Software: J.S., J.M.A., L.Z., I.K. Visualization: I.K., J.S., C.R., J.M.A., R.K., J.B.D., M.C.M. Project administration: J.M.A., R.K. Supervision: J.M.A., R.K., J.S. Writing—original draft: J.M.A., R.K Writing—review and editing: L.Z., J.S., I.K., T.I.M., C.R., J.B.D., M.C.M.

\section{Conflict of Interest}

None declared.

\section{References}

1. Dong E, Du H, Gardner L. An interactive web-based dashboard to track COVID-19 in real time. Lancet Infect Dis 2020;20: 533-34.

2. World Health Organization. The True Death Toll of COVID-19: Estimating Global Excess Mortality. https://www.who.int/data/sto ries/the-true-death-toll-of-covid-19-estimating-global-excess-mor tality (8 June 2021, date last accessed).

3. Dowd JB, Andriano L, Brazel DM et al. Demographic science aids in understanding the spread and fatality rates of COVID-19. Proc Natl Acad Sci USA 2020;117:9696-98.

4. Pifarré I Arolas H, Acosta E, López-Casasnovas G et al. Years of life lost to COVID-19 in 81 countries. Sci Rep 2021;11:3504.

5. Aburto JM, Kashyap R, Schöley J et al. Estimating the burden of the COVID-19 pandemic on mortality, life expectancy and lifespan inequality in England and Wales: a population-level analysis. J Epidemiol Community Health 2021;75:735-40.

6. Karlinsky A, Kobak D. Tracking excess mortality across countries during the COVID-19 pandemic with the world mortality dataset. ELife 2021;10:e69336.

7. Goldstein JR, Lee RD. Demographic perspectives on the mortality of COVID-19 and other epidemics. Proc Natl Acad Sci USA 2020;117:22035-41.

8. Luy M, Di Giulio P, Di Lego V, Lazarevič P, Sauerberg M. Life expectancy: frequently used, but hardly understood. Gerontology 2020;66:95-104.

9. Andrasfay T, Goldman N. Reductions in 2020 US life expectancy due to COVID-19 and the disproportionate impact on the Black and Latino populations. Proc Natl Acad Sci 2021;118. DOI:10.1073/pnas.2014746118.

10. United Nations. World Population Prospects-Population Division-United Nations. https://population.un.org/wpp/ Download/Standard/Population/ (26 January 2021, date last accessed).

11. Aburto JM, Villavicencio F, Basellini U, Kjærgaard S, Vaupel JW. Dynamics of life expectancy and life span equality. Proc Natl Acad Sci USA 2020;117:5250-59.

12. Aburto JM, van Raalte A. Lifespan dispersion in times of life expectancy fluctuation: the case of central and Eastern Europe. Demography 2018;55:2071-96.

13. Ho JY, Hendi AS. Recent trends in life expectancy across high income countries: retrospective observational study. BMJ 2018; $362: \mathrm{k} 2562$.

14. Mehta NK, Abrams LR, Myrskylä M. US life expectancy stalls due to cardiovascular disease, not drug deaths. Proc Natl Acad Sci USA 2020;117:6998-7000.

15. Leon DA, Jdanov DA, Shkolnikov VM. Trends in life expectancy and age-specific mortality in England and Wales, 1970-2016, in comparison with a set of 22 high-income countries: an analysis of vital statistics data. Lancet Public Health 2019;4:e575-82.

16. Fenton L, Minton J, Ramsay J et al. Recent adverse mortality trends in Scotland: comparison with other high-income countries. BMJ Open 2019;9:e029936.

17. Levin AT, Hanage WP, Owusu-Boaitey N, Cochran KB, Walsh SP, Meyerowitz-Katz G. Assessing the age specificity of infection fatality rates for COVID-19: systematic review, meta-analysis, and public policy implications. Eur J Epidemiol 2020;35: 1123-38.

18. Ahrenfeldt LJ, Otavova M, Christensen K, Lindahl-Jacobsen R. Sex and age differences in COVID-19 mortality in Europe. Wien Klin Wochenschr 2021;133:393-98.

19. Hanna TP, King WD, Thibodeau S et al. Mortality due to cancer treatment delay: systematic review and meta-analysis. BMJ 2020;371:m4087. 
20. Wu J, Mamas MA, Mohamed MO et al. Place and causes of acute cardiovascular mortality during the COVID-19 pandemic. Heart 2021;107:113-19.

21. Calderon-Anyosa RJC, Kaufman JS. Impact of COVID-19 lockdown policy on homicide, suicide, and motor vehicle deaths in Peru. Prev Med 2021;143:106331.

22. Jdanov D, Alustiza Galarza A, Shkolnikov V et al. The shortterm mortality fluctuation data series, monitoring mortality shocks across time and space. Sci Data 2021;8:235.

23. Riffe T, Acosta E.; COVerAGE-DB project team. Data resource profile: COVerAGE-DB: a global demographic database of COVID-19 cases and deaths. Int J Epidemiol 2021;50:390-390f.

24. Centers for Disease Control and Prevention. Underlying Cause of Death, 1999-2019 Request. https://wonder.cdc.gov/ucdicd10.html (9 February 2021, date last accessed).

25. ONS. Deaths registered in England and Wales-Office for National Statistics. https://www.ons.gov.uk/peoplepopulatio nandcommunity/birthsdeathsandmarriages/deaths/datasets/ deathsregisteredinenglandandwalesseriesdrreferencetables $(10$ July 2020, date last accessed).

26. Rizzi S, Gampe J, Eilers PHC. Efficient estimation of smooth distributions from coarsely grouped data. Am J Epidemiol 2015; 182:138-47.

27. Pascariu MD, Dańko MJ, Schöley J, Rizzi S. ‘ungroup’: an R package for efficient estimation of smooth distributions from coarsely binned data. J Open Sourc Softw 2018;3:937.

28. ISO week date. Wikipedia. 2021. https://en.wikipedia.org/w/index. php?title=ISO_week_date\&oldid=1007765520 (22 February 2021, date last accessed).

29. Preston SH, Heuveline P, Guillot M. Demography, Measuring and Modeling Population Processes, 1st edn. Oxford: Wiley Blackwell, 2001.

30. Missov TI, Németh L, Dańko MJ. How much can we trust life tables? Sensitivity of mortality measures to right-censoring treatment. Palgrave Commun 2016;2:1-10.

31. Arias E, Tejada-Vera B, Ahmad F. Provisional Life Expectancy Estimates for January through June, 2020. Vital Statistics Rapid Release; no 10. Hyattsville, MD: National Center for Health Statistics, February 2021. DOI: 10.15620/cdc:100392.

32. Silcocks PBS, Jenner DA, Reza R. Life expectancy as a summary of mortality in a population: statistical considerations and suitability for use by health authorities. I Epidemiol Community Health 2001;55:38-43.

33. Horiuchi S, Wilmoth JR, Pletcher SD. A decomposition method based on a model of continuous change. Demography 2008;45: 785-801.

34. Riffe T. DemoDecomp: Decompose Demographic Functions. https://CRAN.R-project.org/package=DemoDecomp $\quad(8$ June 2021, date last accessed).
35. Beltrán-Sánchez H, Preston SH, Canudas-Romo V. An integrated approach to cause-of-death analysis: cause-deleted life tables and decompositions of life expectancy. Demogr Res 2008; 19:1323.

36. Andreev EM, Shkolnikov VM, Begun A. Algorithm for decomposition of differences between aggregate demographic measures and its application to life expectancies, healthy life expectancies, parity-progression ratios and total fertility rates. Demogr Res 2002;7:499-522.

37. Barbieri M, Wilmoth JR, Shkolnikov VM et al. Data resource profile: the Human Mortality Database (HMD). Int J Epidemiol 2015;44:1549-56.

38. Rogers A, Castro LJ. Model Migration Schedules. International Institute for Applied System Analysis Research Report. Austria, 1981, RR-8 1-30.

39. Raymer J, Wiśniowski A. Applying and testing a forecasting model for age and sex patterns of immigration and emigration. Popul Stud (Camb) 2018;72:339-55.

40. Conyon MJ, He L, Thomsen S. Lockdowns and COVID-19 Deaths in Scandinavia. Rochester, NY: Social Science Research Network, 2020.

41. Juranek S, Zoutman F. The Effect of Non-pharmaceutical Interventions on the Demand for Health Care and Mortality: Evidence on COVID-19 in Scandinavia. Rochester, NY: Social Science Research Network, 2020.

42. Avendano M, Kawachi I. Why do Americans have shorter life expectancy and worse health than people in other high-income countries? Annu Rev Public Health 2014;35: 307-25.

43. Lawrence EM, Hummer RA, Domingue BW, Harris KM. Wide educational disparities in young adult cardiovascular health. SSM Popul Health 2018;5:249-56.

44. Faust JS, Krumholz HM, Du C et al. All-cause excess mortality and COVID-19-related mortality among US adults aged 25-44 years, March-July 2020. JAMA 2021;325: 785-87.

45. Glei DA. The US midlife mortality crisis continues: increased death rates from causes other than COVID-19 during 2020. medRxiv 2021; doi: 10.1101/2021.05.17.212 57241.

46. Preston SH, Vierboom YC. Excess mortality in the United States in the 21st century. Proc Natl Acad Sci USA 2021;118; doi: 10.1073/pnas.2024850118.

47. Bambra C, Riordan R, Ford J, Matthews F. The COVID-19 pandemic and health inequalities. J Epidemiol Community Health 2020;74:964-68.

48. R Core Team. R: A Language and Environment for Statistical Computing. Vienna: R Foundation for Statistical Computing, 2020. 